Society for the Anthropology of Work • Book Forum: Threshold

\title{
The Work of Care and \\ Violence
}

Rebecca Richart

Published on: Jul 05, 2021

License: Creative Commons Attribution 4.0 International License (CC-BY 4.0). 
In Threshold: Emergency Responders on the US-Mexico Border, Ieva Jusionyte creatively explores themes of security, violence, health, and borders through an ethnography of the experiences of emergency responders. While this book speaks to multiple areas of interest, reading it as an anthropology of work allow us to identify the ways in which Jusionyte uses the work of emergency responders as an entry point to understand the security apparatus of the U.S.-Mexico border and the terrain on which it operates.

More often than not, Jusionyte allows rich ethnographic descriptions to speak for themselves, as the reader is drawn into the world of emergency responders: their protocols, priorities, and experiences. She moves seamlessly from emergency responders' perspectives on the border to a critique of the very frameworks that define emergency work there. Perhaps the most captivating aspect of Jusionyte's writing is this shifting among the complications, tensions, and conflicts accompanying the performance of care on a violent terrain. In relation to my own research on labor in the U.S. horse racing industry, I recognize the importance of these shifts between understanding work from the perspective of those performing it and zooming out to question the structures that create its very conditions and terms. With this writerly technique, Jusionyte prompts us to examine forms of humanitarian work that avoid larger structural issues and that, in fact, are only necessary because of violent policies and infrastructures.

Throughout the book, Jusionyte reminds us of the importance of studying the infrastructure of a place. What is this infrastructure meant to do, to show, and to obscure? While posing these questions in relation to the border, Jusionyte also explores the daily experiences of individuals working within the constraints and capabilities produced by the border's infrastructure and terrain. With her descriptions of emergency responders' work in the field, we come to understand the textures and materialities of the border in interactions with human bodies. Through her exploration of the binational cooperation of emergency responders, Jusionyte shows the fluidity of the border with respect to capital, utilities, land, water, firefighting strategies, and the articulation of a "greater good." At the same time, she highlights the ways that the border can also function as an obstacle to the cooperative goals of emergency responders.

One of Jusionyte's most chilling points speaks to the ways in which the border is designed as a barrier that "appears innocuous yet is intended to harm" (p. 211). The 
border itself inflicts violence. Emergency responders engage in rescue even as "rescue obscures the politics of wounding" (p. 212). At this pivot point, Jusionyte proposes the concept of the threshold, explaining that it "denotes the juncture between territory and terrain, when an otherwise innocuous constellation of policies and materials achieves the level of toxicity that is harmful to humans" (p. 24). Through this framing, we learn about the life-or-death effects of fluidity and restriction at the border. 